\title{
Dam break over mobile bed: Characterisation of the flow by means of pressure distribution and bed shear stress
}

\author{
Ilaria Fent $^{1}$, Sandra Soares-Frazão ${ }^{1, *}$, and Mario J. Franca ${ }^{2}$ \\ ${ }^{1}$ Institute of Mechanics, Materials and Civil Engineering, Université catholique de Louvain, Belgium. \\ ${ }^{2}$ River Basin Development Chair Group, Water Science \& Engineering department. IHE Delft \\ Institute for Water Education, The Netherlands.
}

\begin{abstract}
The study of the pressure distribution within the dam-break flow over mobile bed is very important because it allows to discuss the hydrostatic pressure distribution, which is the main assumption for the validity of the shallow water equations (SWE). Indeed, this assumption is not always valid, especially when the streamlines are not straight and not perpendicular to the gravity acceleration direction. The flow varies abruptly in the first instants of the falling dam-break wave evolution, but it tires towards a stationary motion during time. To better characterize this phenomenon it is important to examine the evolution of each component of velocity vector field and the distribution of the pressure, which is compared to the hydrostatic distribution. Moreover, the present study considers the distribution of pressure linked with the distribution of the shear stress, through the Navier-Stokes equations. The final object of this work is to characterize the dam break experiment over mobile bed by means of forces acting during the event. The dam-break experiment under examination has been performed in a dedicated flume designed at the laboratory of Civil Engineering Department, Université catholique de Louvain. The analysis of this very transient flow it is now possible and more accurate thanks to the application of non-intrusive new techniques, such as PIV.
\end{abstract}

\section{Introduction}

The consequences caused by a dam break are most of the times catastrophic, in particular if the flow induces the movement of sediment along the valley floor. The measurements on site of this event is practically impossible [1], so laboratory scale experiments reproducing the dam-break flow are required to study in detail its hydrodynamics. Recent studies of the dam-break phenomenon employ the non-intrusive field measurement techniques such as digital imagery, to avoid any obstacle into the flow [2-4]. It is hence possible to measure the velocity distribution, Particle Image Velocimetry [5-8], and to detect water and bed surfaces $[3,4,9]$.

\footnotetext{
* Corresponding author: sandra.soares-frazao@uclouvain.be
} 
Aleixo and others [8] investigated the first stages of the dam-break flow in terms of velocity field, shape of the dam-break wave and evolution towards shallow-water (SW) flow over a fixed bed. They claimed that the vertical component of the velocity becomes negligible after the adimensional time $t^{N D}=2.5$, conforming to the SW assumption [10]. Before reaching the $t^{N D}=2.5$, the flow is still two-dimensional as the vertical velocity and the water surface curvatures play an important role. Thus during the first moments of the dam break, the SW hypothesis are not valid and the streamlines inclination and curvature effects must be accounted for [11]. In this contest we want to make our contribution, in order to study in detail the passage between the non-SW and SW stadium of the flow, accounting also for the distribution of the pressure in space and time. The knowledge of the dam-break flow features has a high degree of interest, in particular because the dam break experimental data are often used as an example of comparison and validation for SW mathematical models.

An added challenge in the study of transient and curved flow is given by mobile bed $[12,13,9]$. The moving sediments on the bed cause a time and space dependent position of the bottom surface, which in turn induce variations in the velocity field [4] and consequently in the pressure distribution. Again this very transient flow over mobile bed is often reproduced numerically following the SW theory accompanied by the morphodynamic formulation. The legitimacy of the 1D numerical models is based on the assumption of hydrostatic distribution of pressure, but during a dam break flow over mobile bed this assumption is not always valid.

Concerning the first instants of the event and just behind the wave front position, the evolution of the bed and the important vertical components of velocity vectors induce important deviation from the hydrostatic pressure, which must be hence investigated [14]. The analysis of the inertial terms that intervene in the distribution of the pressure is actually the principal object of this work. In the calculation of the pressure, we apply a new approach that uses the measured velocity field and flow boundary profiles, evolving in space and time.

The shallow water models involve the use of shear stress formulations, which are used for the computation of external forces acting on the flow and of sediment erosion and deposition. As the flow resistance is a fundamental control of open-channel flows, the investigation of the shear stress on the bed is necessary. In the past a study on the friction effects in unsteady open-channel flows over gravel beds was presented by Tu and Graf [15], where the authors show the evolution of the friction parameter during the passage of a hydrograph. Bathurst [16] examined the flow resistance of high-gradient gravel and boulder-bed rivers and Dressler, Whitham, Hogg and Pritchard [17-19] performed theoretical studies on dam-break flows that show how shear stress affects the velocity field and the front propagation speed. Using the so-called Clauser method, corresponding to the logarithmic law method, we base our analysis of the shear stress on the detection of the longitudinal velocity profile and particularly its logarithmic region.

The purpose of this paper is twofold: we estimate the shear stress and we analyse the pressure distribution in the contest of shallow water hypothesis. We first present the experimental set-up. It follows the estimation of the bed shear stress. Finally, the pressure distribution is analysed and compared with the evolution of the bottom, in order to establish a criterion for the distinction between very near field flow and shallow water flow.

\section{Experimental set-up}

Experiments were performed in a dedicated flume designed at the Universite catholique de Louvain, Civil Engineering Department. The dam-break channel has on overall length of $6 \mathrm{~m}$, and a rectangular cross section defined by a width of $0.25 \mathrm{~m}$ and a 
height of $0.5 \mathrm{~m}$. The channel is divided in two equal reaches by a downward moving gate used to simulate the instantaneous removal of a dam. Fig. 1 shows an overview of the experimental apparatus during the tests. The bottom of the channel is filled with $0.1 \mathrm{~m}$ of non-cohesive sand with the following characteristics: the grain-size distribution is quasiuniform with a mean diameter $d_{50}=1.72 \mathrm{~mm}$, a density $\rho_{s}=2.682 \times 10^{-3} \mathrm{kgm}^{-3}$, a friction angle $\phi=38^{\circ}$ and the bed porosity $\varepsilon_{0}=0.42$. The upstream half of the channel (i.e. the first 3 meters) consisting of the reservoir is filled with a water depth of $h_{0}=0.325 \mathrm{~m}$ over the sand bed.

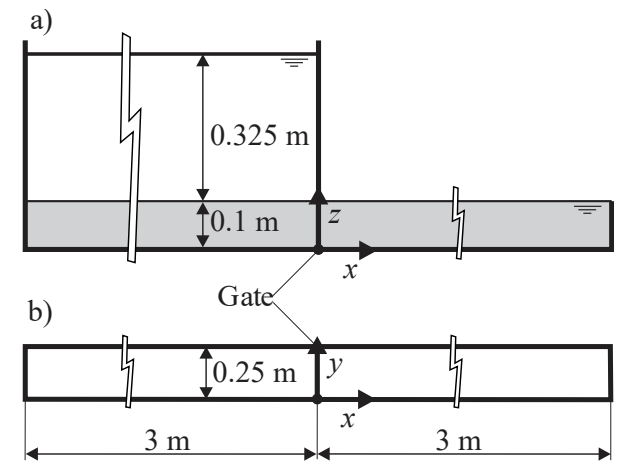

Fig. 1. Dam-break channel at the laboratory of Université catholique de Louvain.

The very transient characteristics of the dam-break flow allow the use of only nonintrusive devices for measurements. Thanks to the use of a very powerful laser and a high speed camera, in this work we apply the light-contrast-based method to identify surfaces [4] and the PIV technology to detect velocity vectors. The camera, which records the event, is positioned in front of the gate and the laser sheet is positioned in the same direction of the flow and $3 \mathrm{~cm}$ far from the transparent wall, so that the images are taken in the longitudinal interval $-0.06 \mathrm{~m}<x<0.15 \mathrm{~m}, \mathrm{y}=0.03 \mathrm{~m}$.

More detailed description of the flume and the measurement technologies are in the $\mathrm{PhD}$ thesis of Fent (2018) and [4].

For the purpose of this paper, 24 runs of the same experiment have been performed and all the variables hereafter proposed are ensemble averaged. Before starting the in-depth analysis, the introduction of some non-dimensional variable is necessary: time $t^{N D}=t \sqrt{g / h_{0}}\left(g\right.$ is the gravity acceleration and $h_{0}$ is the initial water depth upstream the gate); longitudinal and vertical coordinates $x^{N D}=x / h_{0}, z^{N D}=z / h_{0}$; water depth $h^{N D}=h / h_{0}$; horizontal and vertical velocities $u^{N D}=u / \sqrt{g h_{0}}, w^{N D}=w / \sqrt{g h_{0}}$; Shield's parameter of shear stress $\tau^{N D}=\tau /\left[\left(\rho_{s}-\rho\right) g d_{50}\right]$ (water density $\left.\rho=1000 \mathrm{~kg} / \mathrm{m}^{3}\right)$; pressure $p^{N D}=p /\left(g \rho h_{0}\right)$.

\section{Methodologies}

\subsection{Pressure calculation}

When the gate is removed very quickly, a consequent instantaneous adjustment of the pressure occurs. Since the fluid is incompressible, the pressure which was discontinuous upstream and downstream the gate must become continuous on the dam break wave and the adjustment of the pressure causes the resulting unsteady motion [14].

We start by proposing the Navier-Stokes equations that describe the three dimensional motion of a fluid. Considering a two-dimensional motion in the $(x, z)$ plane in a rectangular 
channel, the Navier-Stokes equation for a 2D flow of an incompressible fluid become Eq. (1), written along the vertical direction

$$
\rho\left(\frac{\partial w}{\partial t}+\frac{\partial(u w)}{\partial x}+\frac{\partial w^{2}}{\partial z}\right)=-\frac{\partial p}{\partial z}+\rho v\left(\frac{\partial^{2} w}{\partial x^{2}}+\frac{\partial^{2} w}{\partial z^{2}}\right)-\rho g
$$

Isolating the variation of the pressure term along the vertical direction $\partial p / \partial z$ and integrating the same from the water surface until the random point of vertical coordinate $z$, the pressure at a point $z$ is

$$
p(z)=p_{z_{w}}+\int_{z}^{z_{w}} \frac{\partial p}{\partial z} d z
$$

where $z_{w}$ is the vertical coordinate of free water surface, $p(z)$ is the pressure that depends on the vertical position, $p_{z w}=0$ is the pressure on the free surface.

\subsection{The Clauser method for the calculation of shear stress}

The Clauser method corresponds to the logarithmic law method, based on the analysis of the longitudinal velocity profile and particularly its logarithmic region. First it is necessary to detect the logarithmic region and then to find the logarithmic law that better fits the experimental data. Following Nikuradse [20], the logarithmic low describes the vertical distribution of horizontal velocity $u$ and the shear velocity $u *$ may be obtained from the slope of the best fit line in the inner region. For rough walls the velocity profile is described by Eq. (3).

$$
\frac{u}{u_{*}}=\frac{1}{\kappa} \ln \left(\frac{z+z_{0}}{k_{s}}\right)+B_{r}
$$

where $z_{0}$ is a reference level, $\kappa=0.4$ is the Karman constant, $k_{s}$ is the equivalent sand roughness and $B_{r}$ is the integral constant for rough boundaries. If the wall is completely rough, which corresponds to the condition $u * k_{s} / v>70$, the constant $B_{r}=8.5$ for $z_{0}=0$, according to Nikuradse results.

The slope of the linear trend line found by least square regression for $u$ versus $\ln (z)$ is used to calculate the shear velocity $u *$. Once the shear velocity is calculated it is easy to deduce the shear stress $\tau=\rho(u *)^{2}$, related to the lower point of the logarithmic profile.

\section{Results}

\subsection{Pressure}

The shallow water hypothesis in relation with the pressure distribution are analysed. The time $t^{N D}=0$ was considered as the moment when the gate is completely removed and the end of the recording event is at non-dimensional time $t^{N D}=6.5$.

The results for the velocity field at the very beginning of the dam break (nondimensional time $\left.t^{N D}=0.60\right)$ are shown in Fig. 2. The spatial variation of the horizontal and vertical velocities $\left(u^{N D}\right.$ and $\left.w^{N D}\right)$ is emphasize by the variation in black-white colour scale. The velocity $u^{N D}$ presents higher values near the wave front and increasing values along the vertical direction. The velocity $w^{N D}$ is smaller near the bed and very high for higher water surface curvature and slope. The study of the direction of velocity vectors and the variation 
of the same are very important in order to solve the Eqs. (2) and (3). The velocity vector field is plotted in a polar map (Fig. 3a) and it highlights the important vertical character of the flow at the first instants of dam break. When the flow reaches an almost horizontal direction (Fig. $3 \mathrm{~b}$, at time $t^{N D}=2.50$ ) the vertical component of the velocity is negligible and we can consider that the shallow water character of the flow is reached.

(a)

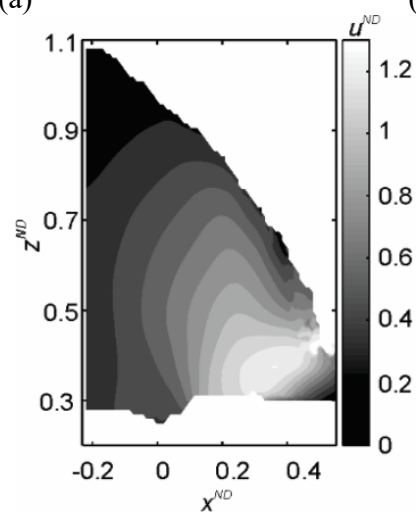

(b)

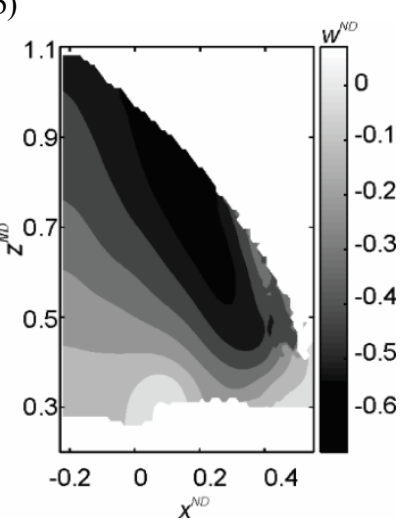

Fig. 2 Velocity field for non-dimensional velocity (a) horizontal component $u^{N D}$ and (b) vertical component $w^{N D}$, at non-dimensional time $t^{N D}=0.60$.

(a)

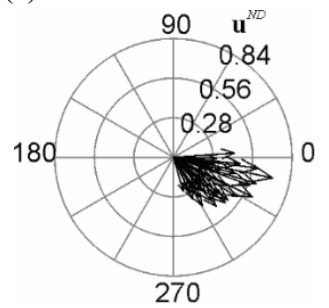

(b)

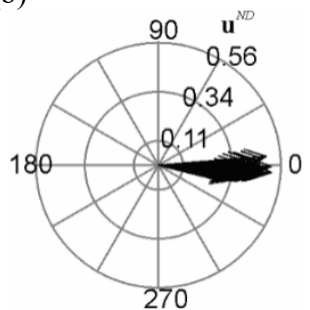

Fig. 3 Velocity vector $\mathbf{u}^{N D}$ distribution at $t^{N D}=0.60$.

(a)

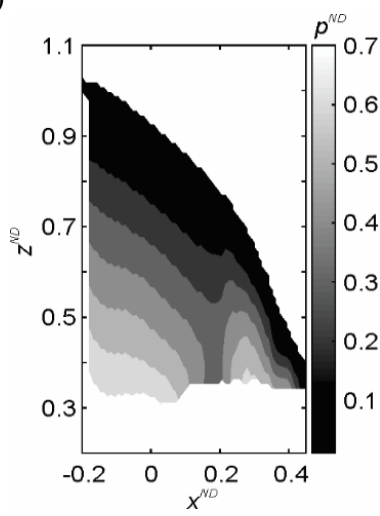

(b)

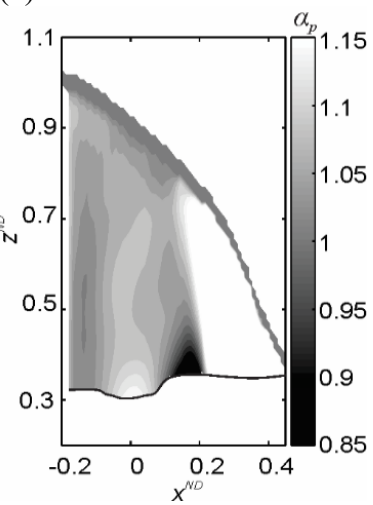

Fig. 4 Pressure at $t^{N D}=0.60$. (a) total pressure and (b) ratio between total and hydrostatic pressure $\alpha_{p}$.

At the same moment $t^{N D}=0.60$, the pressure is calculated and shown in Fig. 4 . The main contribution in the pressure Eq. (2) is given by the gravity dependent terms, while the viscosity terms are very weak. The variation in time and space of the inertial terms is mostly responsible for the deviation from the hydrostatic pressure, in particular on the wave 
front. The configuration of the bed is also responsible of the variation of pressure, as shown in in Fig. 4 . On the depression with positive curvature the total pressure is higher than the hydrostatic one, while on the positive curvature of the bed the total pressure is smaller than the hydrostatic one. At this instant $t^{N D}=0.60$ the pressure calculations confirm the fact that the shallow water hypothesis are not completely valid, as already highlighted by the velocity field, which presents an important vertical velocity component (Fig. 3a).

The evolution in space and time of the ratio $\alpha_{p}$ between the total and hydrostatic pressures at the bed level is illustrated in Fig. 5. It is clear that in the first instants the total pressure remains very different from the hydrostatic pressure, in particular at the front of the wave. After $t^{N D}=3.50$, the flow reaches the shallow water condition and the deviation of total pressure from the hydrostatic pressure is negligible.

\subsection{Shear stress}

The calculation of the shear stress is done by means of Eq. (3). During dam break experiments over mobile sand bed, only sediments in the upper layer of the bed are transported and change their position in space and time, the rest of sediments do not move. Because of that, we decide to impose the vertical reference level zero at $z=8.5 \mathrm{~cm}$ over the fixed bed (see some examples in Fig. 6).

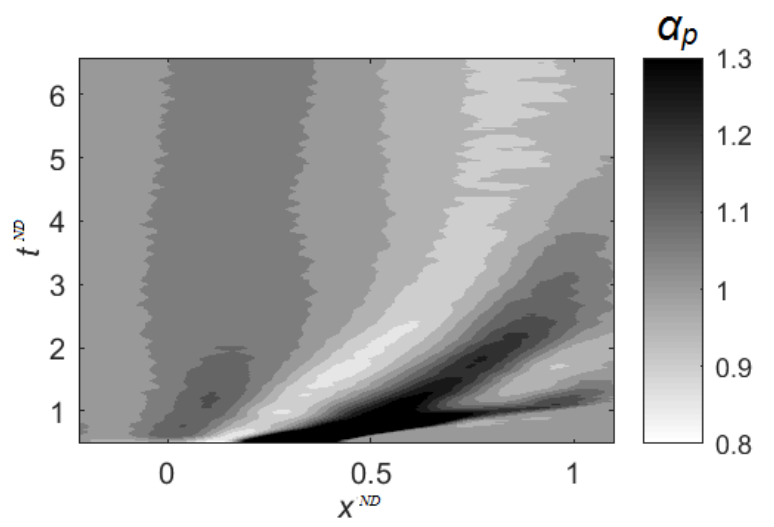

Fig. 5 Ratio between total and hydrostatic pressure, function of space and time.

The horizontal velocity profiles are calculated after ensemble (24 runs) averaging the values of horizontal velocity. Within the clear water layer (over the mobile bed subject to bed load transport) the velocity profiles are plotted in a semi-logarithmic graph (Fig. 6) with $x$ coordinate equal to $\ln (z)$ and $y$ coordinate equal to $u$. Eq. (3) is represented by a straight line of which the slope is given by $\mathrm{u}^{*} / \kappa$. In this graph we decide to keep the dimensional variables, in order to better extrapolate the final interesting value of the line slope. We can distinguish for each subfigure of Fig. 6 two different zones with two different line slopes, which correspond to two shear velocities. Consequently it is possible to calculate two shear stresses that act on the sediment bed $\left(\tau_{\mathrm{s}}\right)$ and on the limit of the boundary layer $\left(\tau_{\mathrm{w}}\right)$.

The two non-dimensional shear stresses (Shield's parameters) are plotted for several times and at different positions along the channel (Fig. 7). In Fig. 7a it is visible that the bed shear stress $\left(\tau_{\mathrm{s}}\right)$ increases in time but remains almost constant in space. Fig. $7 \mathrm{~b}$ shows the calculated shear stress relative to the smaller slope of the trend lines in Fig. 6, representing the semi-logarithmic profile of horizontal velocity in the main section of clear water layer. In this portion of the profile the velocity is almost constant, so the slope of the interpolated line is very small. The shear stress on the clear water layer is very weak because the 
dam break is a very transient flow and doesn't allow the immediate development of the boundary turbulent layer.
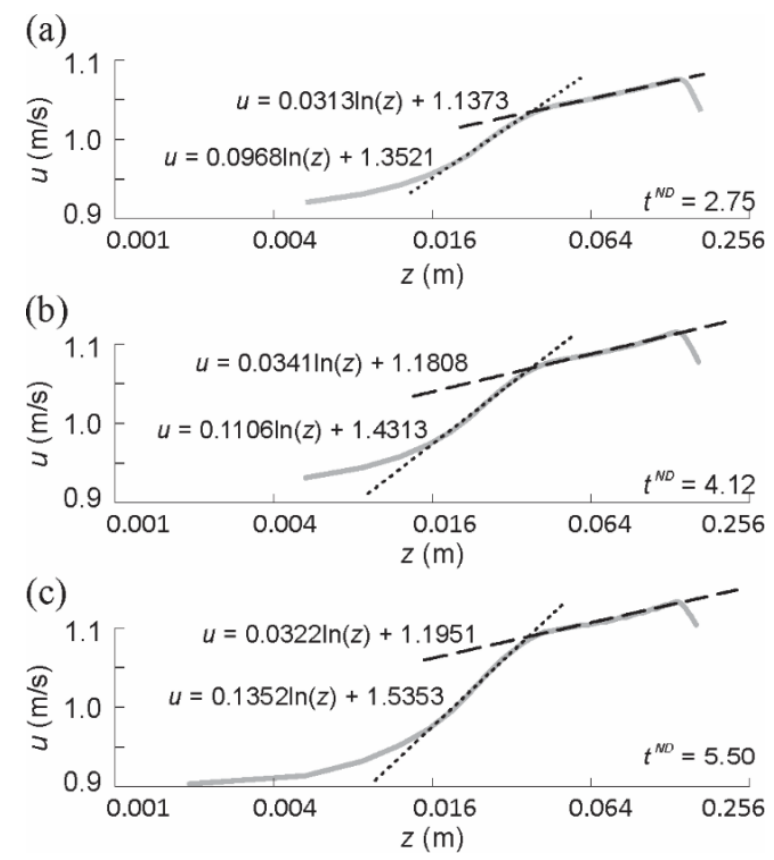

Fig. 6 Semi-logarithmic profiles of horizontal velocity $u$, at non-dimensional times (a) $t^{N D}=2.75$, (b) $t^{N D}=4.12$, (c) $t^{N D}=5.50$, on the section $x^{N D}=0$.

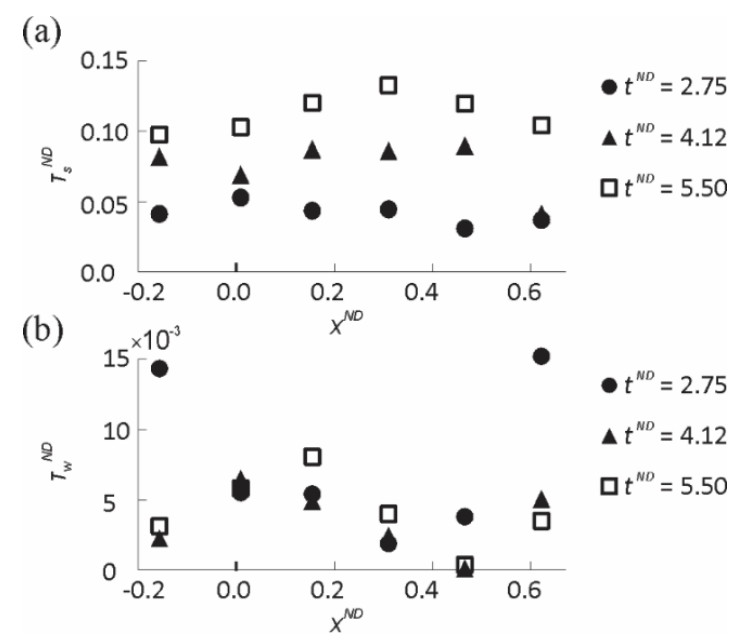

Fig. 7 Shield's parameter calculated by using the logarithmic law (3): (a) over the sediment layer $\tau_{\mathrm{s}}^{N D}$ and (b) over the boundary layer $\tau_{\mathrm{w}}{ }^{N D}$.

\section{Conclusion}

The new dam-break flow experiments over a mobile bed made of coarse uniform sand were conducted with a special focus on the pressure distribution and the shear stress. Using the 
image treatment technique and the PIV approach, the hydraulic variables as the velocity and the water and bed surfaces were measured and used for the forward analysis. 24 runs of the same experiment of dam break are performed and images are recorded on the gate zone, where the phenomenon starts.

The two dimensional velocity distribution is used to calculate the pressure, varying in space and time. We detect important deviation from the hydrostatic pressure during the first instants of the event, while after $t^{N D}=3.5$ the flow is almost hydrostatic and the shallow water hypothesis are hence effective. This result is significant when the dam break over mobile bed is simulated by shallow water numerical model. The same models involve the use of shear stress formulations, which are typically relating to the uniform steady flow. The investigation of the shear stress for high transient flow over mobile bed is hence very important and here the bed shear stress is investigated. The bed shear stress is calculated by the means of shear stress velocity extrapolated by the semi-logarithmic velocity profile. The profile shows a peculiar aspect of the slope: the presence of two different slopes of the logarithmic law, generally higher near the bed and smaller on the upper part of the water depth, brings to two different values of shear velocity and consequently shear stress.

\section{References}

1. H. Chanson. Second Edition. Elsevier Butterworth-Heinemann, Oxford (2004).

2. S. Soares-Frazão. J. of Hydr. Res. 45:sup1, 19-26 (2007).

3. R. Aleixo, B. Spinewine, S. Soares-Frazão, Y. Zech. Proceedings of the 33rd IAHR Conference, Vancouver, pp:6898-6905 (2009).

4. I. Fent, S. Soares-Frazão, Y. Zech. Dam-break flow experiments over mobile bed: velocity profile. Accepted in J. Hydr. Res. (2018)

5. R. Keane, R. Adrian. Applied Science Research 49, 191-215 (1992).

6. M. Raffel, C. Willert, S. Werely, J. Kompenhans. Springer-Verlag (2007).

7. R.J. Adrian, J. Westerweel. Cambridge University Press (2011).

8. R. Aleixo, S. Soares-Frazão, Y. Zech. Experiments in fluids, 50(6), 1633-1649 (2011).

9. B. Spinewine, H. Capart. Journal of Fluid Mechanics, 731, 579-614 (2013).

10. J. Stoker. Water Waves: New York: Wiley-Interscience (1957).

11. W.H. Hager, K. Hutter. Acta Mechanica, 51(1-2), 31-48 (1984).

12. R. Aleixo, B. Spinewine, S. Soares-Frazão, Y. Zech. In Proceedings of the RiverFlow 2010 conference, Braunschweig, Germany (2010).

13. O. Castro-Orgaz, W.H. Hager. J. Hydr. Res. 51(2), 203-208 (2013).

14. P. Stansby, A. Chengini, T. Barnes. J. Fluid Mech. 374, 407-424 (1998).

15. H. Tu, W.H. Graf. J. of Hydr. Res., 31(1), 99-110 (1993).

16. J.C. Bathurst. J. Hydr Eng. 111(4), 625-643 (1985).

17. R.F. Dressler. National Bureau of Standards (1952).

18. G.B. Whitham. In Proceedings of the Royal Society of London A: Mathematical, Physical and Engineering Sciences, 227 (1170), 399-407 (1955, January).

19. A.J. Hogg, Pritchard D. J. Fluid Mech. 501, 179-212 (2004).

20. J. Nikuradse. In VDI Forschungsheft (1933). 